\title{
Proposal for a Datta-Das transistor in the quantum Hall regime
}

\author{
Luca Chirolli, Davide Venturelli, Fabio Taddei, Rosario Fazio, and Vittorio Giovannetti ${ }^{1}$ \\ ${ }^{1}$ NEST, Scuola Normale Superiore and Istituto Nanoscienze-CNR, I-56127 Pisa, Italy
}

\begin{abstract}
We propose a resonant spin-field-effect transistor for chiral spin-resolved edge states in the integer quantum Hall effect with Rashba spin-orbit interaction. It employs a periodic array of voltagecontrolled top gates that locally modulate the Rashba spin-orbit interaction. Strong resonant spinfield-effect is achieved when the array periodicity matches the inverse of the wave-vector difference of the two chiral states involved. Well known techniques of separately contacting the edge states make possible to selectively populate and read-out the edge states, allowing full spin read-out. The resonant nature of the spin-field effect and the adiabatic character of the edge states guarantee a high degree of robustness with respect to disorder. Our device represents the quantum Hall version of the all-electrical Datta-Das spin-field effect transistor.
\end{abstract}

PACS numbers:

\section{INTRODUCTION}

Research in spintronics 1 , a spin based electronics, has led to a number of fundamental discoveries in physics and it has a clear potential impact in the emerging quantum technologies, such as quantum information processing 2 . Among the most intriguing proposals in this context there is the spin-field-effect transistor (SFET) introduced by Datta and Das (for a review see also Ref. [1] and references therein). Spin-polarized carriers are selectively injected in a two-dimensional electron gas (2DEG), while spin precession is externally controlled by means of a local gate voltage which modulates the Rashba SpinOrbit Interaction (SOI) 4 . The possibility of achieving gate control of the SOI strength has been experimentally established since the end of the '90s - see e.g. Refs. [68. Spin-polarized injection into a semiconductor proved instead to be a more difficult task ${ }^{19}$ which has been partially overcome only recently 10 delaying the first implementation of a SFET by almost a decade 11 .

The challenging requirements of the SFET can be more naturally met in a quantum Hall system. In the present work we introduce a generalization of the DattaDas transistor in which gate-controlled spin procession is accompanied by coherent electron transfer between copropagating chiral edge channels of the integer quantum Hall effect (IQHE) ${ }^{12}$. Due to the possibility of individually contacting chiral edge states that have opposite values of the spin degree of freedom, our proposal completely avoids the injection problems $\frac{910}{10}$ one faces in the original SFET setting. Furthermore, our implementation of the SFET represents a useful tool available for quantum Hall interferometry 13 , since it can also be employed as a tunable beam-splitter, especially in the newly introduced co-propagating architecture of Ref. [16. As such it constitutes a potentially scalable building block for implementing quantum information processing $17+19]$ based on dual-rail encoding schemes 20 . Finally, chiral states of the IQHE are ideal for long distance transfer of spin states, owing to the very large relaxation ${ }^{21 \mid 22}$ and coherence ${ }^{13}$ lengths.

The quantum Hall SFET discussed here operates in the

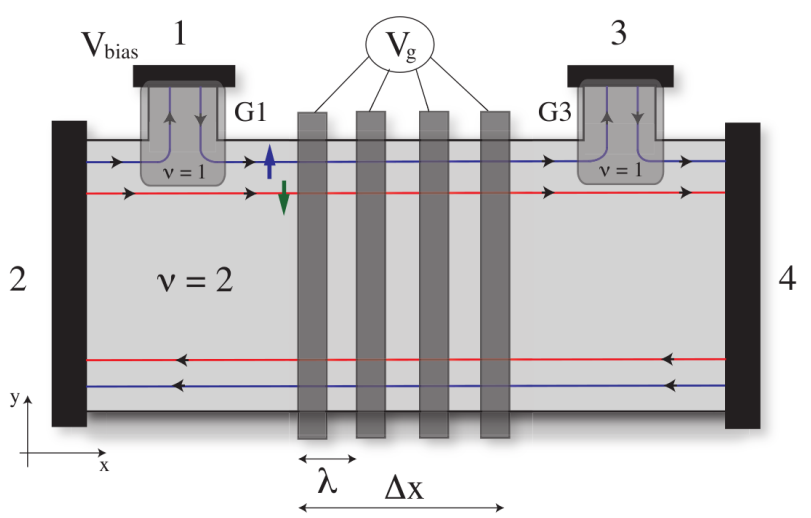

FIG. 1: (Color online) Schematics of the setup: black and gray elements are contacts and top gates, respectively, while the chiral edge states are represented by blue (outer edge $\psi_{o}$, spin-up) and red (inner edge $\psi_{i}$, spin-down) trajectories. The periodic array of top gates controlled by the voltage $V_{g}$ represents the scattering active region.

IQHE regime at filling factor $\nu=2$ (number of filled energy levels in the bulk) in the presence of a weak uniform Rashba SOI. The two orthogonal chiral states $\psi_{o}$ and $\psi_{i}$ belonging to the lowest Landau level co-propagate at slightly different wave vectors, $k_{o}$ and $k_{i}$, spatially separated along the transverse $y$-direction, and have (almost) opposite $z$-spin components. The underlying principle of operation can be summarized as follows (the setup is schematized in Fig. 1). Exploiting large top gates G1 and G3 that locally lower the filling factor to $\nu=1$, it is possible to separately contact $\psi_{o}$ and $\psi_{i}$. Specifically, spin-up electrons (in $\psi_{o}$ ) can be injected by applying a bias voltage $V_{\text {bias }}$ to contact 1 , leaving the other three contacts grounded, while a measurement of the charge current in contact 3 and 4 allows to determine the spin in a spin-charge conversion fashion. Spin precession occurs if the difference $\Delta k=k_{o}-k_{i}$ in momentum of the two chiral states is compensated (this is not required in the original Datta and Das setting). To tackle this problem we adopt a periodic-poling technique similar to 
that one experimentally realized in Ref. 22] where an engineered coupling between co-propagating chiral spinresolved edge states was achieved by means of an array of magnetic Cobalt contacts.

Specifically, we consider a periodic array of electric top gates, placed between contacts 1 and 3 of the device (see Fig. 1), which generate a periodic electrostatic potential and modulate the Rashba SOI in the 2DEG region underneath them. Under these conditions, we show that when the periodicity $\lambda$ of the modulation is adjusted at the resonant condition $\lambda_{\text {res }}=2 \pi / \Delta k$ a strong enhancement of the spin transfer is obtained and it can be externally controlled by varying the top gate voltage (typical values of $\lambda_{\text {res }}$ are hundreds of nanometers in GaAs, easily experimentally achievable - see e.g. Refs. 22/23]). As a result, in the same spirit of the original Datta and Das scheme ${ }^{3}$, our method provides an electrically tunable way to achieve a resonant spin-field-effect transistor in quantum Hall systems.

The article is structured as follows. In Sec. Пwe introduce the model and define its Hamiltonian. In Sec. III we address the transport properties of the system. In Sec. IV we test the robustness of the device in the presence of disorder. Finally we conclude in Sec. V

\section{THE MODEL}

A rigorous analysis of the effect is obtained by expressing the chiral edge states $\psi_{o}$ and $\psi_{i}$ as eigenvectors of the unperturbed Hamiltonian $H=H_{0}+H_{R}$, where

$$
H_{0}=\frac{\Pi^{2}}{2 m^{*}}+V_{c}(y)-\frac{E_{z}}{2} \sigma_{z},
$$

describes a 2DEG in the presence of a strong perpendicular magnetic field $B$ and of a transverse confining potential $V_{c}(y)$, and

$$
H_{R}=\alpha\left(\Pi_{x} \sigma_{y}-\Pi_{y} \sigma_{x}\right),
$$

is the weak uniform Rashba SOI contribution. The Rashba coupling constant is denoted by $\alpha$, while $\sigma_{x, y, z}$ are the spin Pauli matrices. $\boldsymbol{\Pi}=-i \hbar \boldsymbol{\nabla}+e \mathbf{A}$ is the $2 \mathrm{D}$ kinetic momentum in the Landau gauge $\mathbf{A}=(-B y, 0,0)$, $E_{z}=g^{*} \mu_{B} B$ the Zeeman gap, $m^{*}$ and $g^{*}$ the effective mass and $g$-factor of the material, respectively. The effect of a uniform Rashba SOI in the IQHE has been intensely studied in the past 2427 . The eigenstates $\psi_{o}$ and $\psi_{i}$ are given in terms of dressed states composed by linear combination of adjacent Landau levels of opposite spin 24. Defining the cyclotron gap $E_{c}=\hbar \omega_{c}$, with $\omega_{c}=e B / m^{*}$ the cyclotron frequency, and the magnetic length $\ell_{B}=\sqrt{\hbar / e B}$, at first order in $\hbar \alpha / \ell_{B} E_{c}$ the edge states are

$$
\begin{aligned}
& \psi_{o} \approx \psi_{0, k_{o}, \uparrow}+i \frac{\sqrt{2} \hbar \alpha}{\ell_{B}\left(E_{c}+E_{z}\right)} \psi_{1, k_{o}, \downarrow} \\
& \psi_{i}=\psi_{0, k_{i}, \downarrow}
\end{aligned}
$$

where $\psi_{n, k, \sigma}$ are the eigenvectors of $H_{0}$ associated with the $n$-th Landau level, longitudinal momentum $k$, and having spin $z$-projection equal to $\sigma$. Under the assumption of smooth confinement $V_{c}(y)$, the latter can be written as $\psi_{n, k, \sigma}(x, y) \simeq e^{i k x} \chi_{n}\left(y-k \ell_{B}^{2}\right)|\sigma\rangle / \sqrt{L}$, with $\chi_{n}(y)$ being the $n$-th eigenfunction of the harmonic oscillator and $L$ is the length of the Hall bar in the $x$-direction. In a single-particle picture, the values $k_{o}$ and $k_{i}$ can be estimated from the degeneracy condition $V_{c}\left(k_{o, i} \ell_{B}^{2}\right)-m^{*} \alpha^{2}(1 \pm 1) /\left(1+E_{z} / E_{c}\right) \mp E_{z} / 2=E_{F}-E_{c} / 2$, where $E_{F}$ is the Fermi energy of the system, the ratio $E_{z} / E_{c}=m^{*} g^{*} \mu_{B} / e \hbar$ being a material-dependent constant. The momenta difference is $\Delta k=k_{o}-k_{i} \simeq$ $\left(E_{z}+m^{*} \alpha^{2} /\left(1+E_{z} / E_{c}\right)\right) /\left.\left(\ell_{B}^{2} \partial_{y} V_{c}\right)\right|_{E_{F}}$, and the transverse spatial separation of the edge states is $\Delta y=\Delta k \ell_{B}^{2}$. The latter is substantially renormalized by exchange interaction ${ }^{28}$, which we effectively take into account in the renormalization of the $g$-factor. According to this analysis the $\psi_{o}$ edge state will carry essentially spin-up electrons at momentum $k_{o}$, while $\psi_{i}$ purely spin-down electrons at momentum $k_{i}$.

\section{A. Modulated Rashba SOI}

The perturbation introduced by the top gate array of Fig. 1] (which extends in the longitudinal $x$-direction for a region of length $\Delta x \ll L$ ) produces two net effects, both contributing to electron transfer from $\psi_{o}$ to $\psi_{i}$. First of all, the array of top gates induces a periodic modulation of the electrostatic potential underneath the gates that can be described by adding to the Hamiltonian a term

$$
U_{m}(x)=U\left(V_{g}\right) \cos ^{2}(\pi x / \lambda) .
$$

The latter locally mixes the Landau levels and it can open mini-gaps in the spectrum. However, as long as the potential $U_{m}$ is restricted to a small region $\Delta x$ and $U\left(V_{g}\right) \ll E_{c}$, no qualitative change is expected in the structure of edge states 29 . In this regime, even though $U_{m}$ does not couple to the spin degree of freedom of the system, it can contribute indirectly to the coupling between $\psi_{o}$ and $\psi_{i}$ due to the modification of the edge states induced by the homogeneous $H_{R}$ term in the Hamiltonian. Furthermore, a second important effect introduced by the gate array is a periodic modulation of the Rashba coupling constant $\alpha$. We describe it as an additional perturbation term to $H$ of the form

$$
\delta H_{R}(x)=\frac{1}{2}\left\{\delta \alpha(x), \Pi_{x}\right\} \sigma_{y}-\delta \alpha(x) \Pi_{y} \sigma_{x},
$$

where the modulation $\delta \alpha(x)=\delta \alpha\left(V_{g}\right) \cos ^{2}(\pi x / \lambda)$ has the same spatial dependence as $U_{m}(x)$ but strength $\delta \alpha\left(V_{g}\right)$, and where the symmetrized product $\left\{\delta \alpha(x), \Pi_{x}\right\} / 2=\left(\delta \alpha(x) \Pi_{x}+\Pi_{x} \delta \alpha(x)\right) / 2$ is inserted to ensure hermiticity of the SOI term. Similar contributions have been analyzed for spin transport in quantum wires, both in a single particle-picture ${ }^{30}$ and for an interacting nanowires ${ }^{31}$ and helical modes in the quantum spin Hall effect ${ }^{32}$. The $\delta H_{R}(x)$ term provides a 
direct spin-flip mechanism that is resonantly enhanced by the periodicity of the gate array and couples $\psi_{o}$ and $\psi_{i}$. According to Refs. 6[11, $\alpha$ is approximatively a linear function of $V_{g}$ in InAs-based heterostructures. More precisely, for a large top gate $\hbar \alpha$ varies within $[0.7,0.9] \times 10^{-11} \mathrm{eVm}$ in the voltage range $V_{g}=[0,-1]$ $\mathrm{V} 6$, and $\hbar \alpha \sim[0.9,1.3] \times 10^{-11} \mathrm{eVm}$ in the voltage range $V_{g}=[0,-3]$ V11. It follows that $\delta \alpha\left(V_{g}\right)$ can be tuned almost by a factor 2 and that $\delta \alpha\left(V_{g}\right) / \alpha \lesssim 1$ for all practical purposes.

\section{TRANSPORT}

We characterize the transport properties of the system in the Landauer-Büttiker formalism 33 . The current flowing through the output contacts can be determined knowing the amplitudes for scattering off the region underneath the top gate array of Fig. 1. Asymptotic states are defined far away from the scattering region and are given by the edge states of the Hall bar $\psi_{o}$ and $\psi_{i}$, Eqs. (34). The scattering amplitudes, at the Fermi energy $E_{F}$, can be generally expressed in terms of the matrix elements of the operator $T=\Delta H+\Delta H G^{+}\left(E_{F}\right) \Delta H, \frac{34}{,}$ where $G^{+}(\epsilon)=\left(\epsilon+i 0^{+}-H-\Delta H\right)^{-1}$ is the full retarded Green's function of the system, $\Delta H=U_{m}(x)+\delta H_{R}(x)$ is the scattering potential (finite only in a region of length $\Delta x$ ) and $H+\Delta H$ is the overall Hamiltonian of the system. In particular, the transmission amplitude for scattering from the incoming state $\psi_{i}$ to the outgoing states $\psi_{o}$ is given by $t_{i o}=\left(L / i \hbar \sqrt{\left|v_{i}\right|\left|v_{o}\right|}\right)\left\langle\psi_{o}|T| \psi_{i}\right\rangle$, where $v_{i}$ and $v_{o}$ are the velocities of the incoming and outgoing states, respectively.

Let us assume first that the term $\Delta H$ can be treated as a perturbation with respect to $H$ (i.e. an assumption valid for small $V_{g}$ ). Setting $v_{i}=v_{o} \equiv v_{D}=$ $\partial V_{c}\left(k \ell_{B}^{2}\right) /\left.\partial \hbar k\right|_{E_{F}}$ the drift velocity of the system, we can evaluate the scattering amplitude $t_{i o}$ in the Born approximation $t_{i o} \simeq\left(L / i \hbar v_{D}\right)\left\langle\psi_{i}|\Delta H| \psi_{o}\right\rangle$ and we find

$$
t_{i o}=t\left(V_{g}\right) \operatorname{sinc}((\Delta k-2 \pi / \lambda) \Delta x / 2),
$$

where $\operatorname{sinc}[\cdot]=\sin [\cdot] /[\cdot]$ is the cardinal sinus, and where

$$
t\left(V_{g}\right)=\frac{\Delta x \Delta k}{2 v_{D}}\left[i \delta \alpha\left(V_{g}\right)-\frac{U\left(V_{g}\right)}{E_{c}+E_{z}} \alpha\right] e^{-\frac{\Delta k^{2} \ell_{B}^{2}}{4}} .
$$

This is the main result of the paper. Equation (7) clearly shows how the periodicity of the gate array can provide the correct amount of momentum transfer giving rise to resonant coupling of the edge states at $\lambda=\lambda_{\text {res }}$. Equation (8) instead confirms that both $U_{m}(x)$ and $\delta H_{R}(x)$ contribute to the coupling and shows that, as in the original Datta-Das spin-field transistor, the intensity of the coupling can be tuned electrically with the gate voltage $V_{g}$ [both $U\left(V_{g}\right)$ and $\delta \alpha\left(V_{g}{ }^{\sqrt{6111}}\right.$ can be taken as linear function of $V_{g}$ ]. Interestingly, the two contribution in Eq. (8) are $\pi / 2$ phase-shifted and they cannot cancel. This can be understood noticing that the origin of the

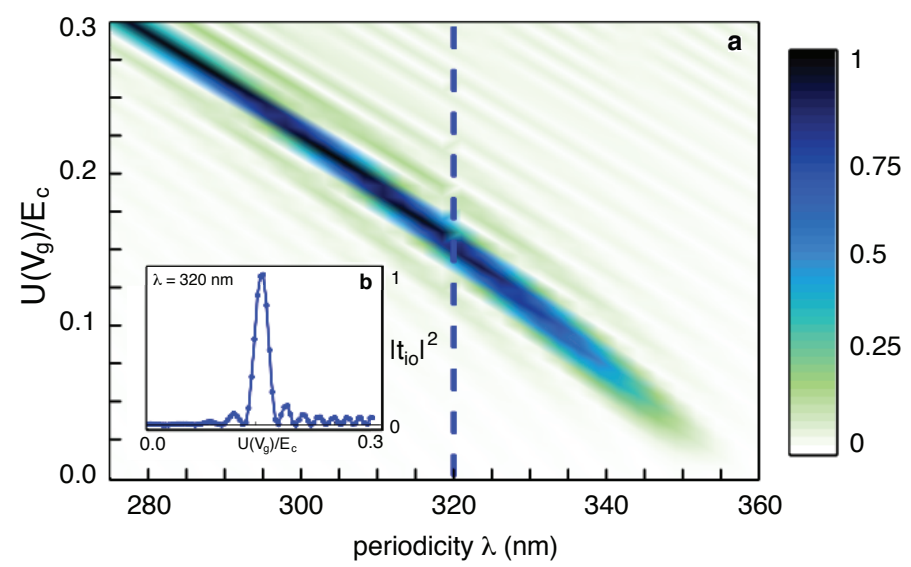

FIG. 2: (Color online) a) Density plot of $\left|t_{i o}\right|^{2}$ versus the array periodicity $\lambda$ and the amplitude $U\left(V_{g}\right)$ of the modulation potential. Both uniform and modulated Rashba SOI, ( $\alpha$ and $\left.\delta \alpha\left(V_{g}\right)\right)$ are present, as detailed in the text. The total number of gates is 50 independently from $\lambda$ : the abscissa spans a modulation length $\Delta x$ that ranges from $12.5 \mu \mathrm{m}$ to $18 \mu \mathrm{m}$. b) $\left|t_{i o}\right|^{2}$ corresponding to $\lambda=320 \mathrm{~nm}$ (dashed line in the density plot). These results are obtained by means of the scattering formalism applied to the system shown in Fig. 1, described on a lattice by means of the KNIT open source package ${ }^{35}$.

interplay between the electrostatic modulation potential and the uniform Rashba SOI arises from their non-zero commutator, $\left[H_{R}, U_{m}\right] \propto \partial_{x} U_{m} \sigma_{y}$, that turns out to be oscillating and $\pi / 2$ phase-shifted with respect to $U_{m}$ and to $\delta H_{R}(x)$.

The results obtained above are valid in the perturbative regime of weak Rashba SOI $\left(\hbar \alpha / \ell_{B} E_{c}=\ell_{B} / \ell_{\text {so }} \ll 1\right.$, where the spin-orbit length $\ell_{\mathrm{so}}=\hbar / m^{*} \alpha$ characterizes the scale of the SOI) and weak Zeeman energy $\left(E_{z} / E_{c} \ll 1\right)$. For GaAs-based heterostructures such conditions are widely fulfilled. For InAs the spin-orbit length can be estimated to be of the order of hundreds on nanometers (using $m^{*}=0.02 m_{0}$ ) and, for $B \sim 5 \mathrm{~T}$, one has $\ell_{B} / \ell_{\text {so }} \sim 0.1$. The Zeeman energy is also relatively large due to the large $g$-factor, $g_{\mathrm{InAs}}^{*} \sim 3-10$ so that $E_{z} / E_{c} \sim 0.1$. Therefore, a perturbative analysis is relevant.

\section{A. Numerical Analysis}

We have checked the validity of the analytical perturbative approach by numerical calculations based on a tight-binding Hamiltonian describing the system in Fig. 1. Transport properties are computed through the recursive-Green's function algorithm of the KNIT Numerical Package ${ }^{35}$, which allows to compute local and non-local observables on tight-binding Hamiltonians $12 \mid 36$. The total transmission between lead 1 and lead 4 of the system (see Fig. 1) can be obtained from the retarded Green's functions through the Fisher-Lee rela- 


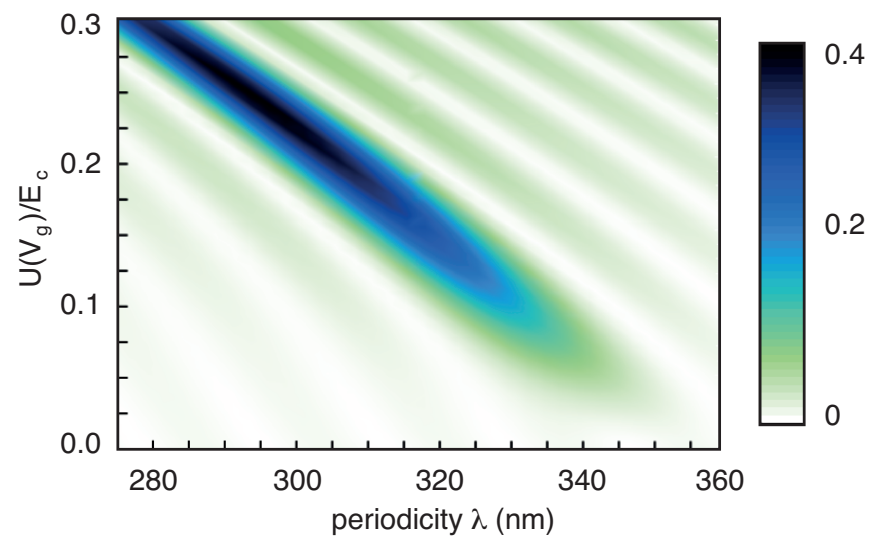

FIG. 3: (color online) Density plot of the transmission probability $\left|t_{i o}\right|^{2}$ versus applied modulation potential $U\left(V_{g}\right)$ and array periodicity $\lambda$, for an array of 20 top gates. A maximum transmission probability of $40 \%$ is achieved for a periodicity $\lambda \sim 290 \mathrm{~nm}$ for suitable applied voltage producing on the 2DEG a potential $U\left(V_{g}\right) \sim 0.25 E_{c}$.

tions 37 . The leads are assumed with no SOI. The width of the Hall bar is taken to be $100 \mathrm{~nm}$ (hard wall confining potential), discretized on a square lattice of spacing 2.5 $\mathrm{nm}$. The external magnetic field is taken to be $6 \mathrm{~T}$, corresponding to a cyclotron gap $\hbar \omega_{c} \approx 34.7 \mathrm{meV}$ for InAs, and it is introduced by means of Peierls phase factor. The Zeeman gap has been taken to be $E_{\mathrm{Z}}=\hbar \omega_{c} / 15$, corresponding to an effective value of $g^{*}=6.6$. This value is of the order of magnitude of the exchange-enhanced value measured in InAs devices in the quantum Hall regime 38 .

Fig. 2 a shows the transmission probability $\left|t_{i_{o}}\right|^{2}$, in a density plot, as a function of the periodicity $\lambda$ and the amplitude $U\left(V_{g}\right)$ of the potential, for a total fixed number of top gates equal to 50. In the simulations we set $\hbar \alpha=3 \times 10^{-12} \mathrm{eVm}$, and simultaneously vary $\delta \alpha\left(V_{g}\right)$ in the range $[0, \alpha]$, and $U\left(V_{g}\right)$ in the range $\left[0, \hbar \omega_{c} / 3\right]$. The Fermi energy is taken in the middle of the $\nu=2$ plateau $\left(E_{F}=E_{c}\right)$, so that even for the maximum value of $U\left(V_{g}\right)$ backscattering is absent. For these parameters, in the absence of the array we obtain an intrinsic channel mixing of less than $0.1 \%$ (due to the broken translation invariance owing to the geometry of the injection/detection regions in the device), which confirms that the effect of SOI, as such, is negligible.

The plot shows a full resonant line in the $\lambda-U\left(V_{g}\right)$ plane in which spin flip is achieved. For moderate value of $U\left(V_{g}\right)$ the transmission probability reaches unity and complete spin-flip accompanied by total charge transfer is atteined. Beside the resonance line, a series of secondary peaks appears, which can be understood as interference fringes in the framework of the perturbative analysis, Eq. (7). For increasing values of the electrostatic potential $U\left(V_{g}\right)$ we see a shift of the resonant periodicity $\lambda_{\text {res }}$ to smaller values. This is consistent with a local non-linear modification of the confining potential and a local decrease of the Fermi energy, together yielding an increase of the wave vector difference, which acquires an effective gate-voltage dependence, $\Delta k\left(V_{g}\right)$, entailing a "red-shift" of the resonant peak $\lambda_{\text {res }}=2 \pi / \Delta k$. Since the value of the resonant periodicity is a priori unknown and sample-dependent, such effect turns out to be a useful control knob of the setup (the range of periodicities at which a given sample can resonate spanning more than $100 \mathrm{~nm}$ ). As shown in Fig. 2b for a fixed periodicity, by varying the voltage applied to the gates it is possible to control the precession of the spin, characteristic of a SFET. We also note that the external magnetic field represents an additional tuning parameter that is expected to affect the resonance condition.

For the sake of completeness we present in Fig. 3 results for an array of 20 top gates. The overall response presents no qualitative changes, but a smaller maximum transmission probability and interference fringes with larger periodicity, consistent with the reduced number of top gates. We point out that the characteristics of a transistor is a high/low current response with respect to a threshold. Therefore, also in the case of an array with 20 top gates shown in Fig. 3 a good transistor functionality can be achieved.

\section{B. Dresselhaus SOI}

It is worth noticing that in III-V compounds the Dresselhaus SOI, originating from a bulk inversion asymmetry in zinc-blende structures ${ }^{42}$, is also present. When restricted to quantum wells, by aligning our reference frame to the cubic crystallographic axes such that $x, y$, and $z$ correspond to the [100], [010] and [001], respectively, (with [001] the heterojunction growth direction) and neglecting cubic corrections in the momentum, the Dresselhaus Hamiltonian reads

$$
H_{D}=\beta\left(\Pi_{x} \sigma_{x}-\Pi_{y} \sigma_{y}\right),
$$

with coupling constant $\hbar \beta \simeq 27(\pi / d)^{2} \mathrm{eV}^{3}$ for both GaAs and InAs $24, d$ being the width of the quantum well. In InAs, in which the fundamental gap is smaller than GaAs, the Rashba term dominates, whereas in GaAs, Dresselhaus and Rashba SOIs have comparable strength and both contribute to the modification of the edge states. In the perturbative regime $\alpha, \beta \ll \omega_{c} \ell_{B}$ this can be easily taken into account: at first order in $H_{R}+H_{D}$ the edge states are

$$
\begin{aligned}
& \psi_{o} \approx \psi_{0, k_{o}, \uparrow}+i \frac{\sqrt{2} \hbar \alpha}{\ell_{B}\left(E_{c}+E_{z}\right)} \psi_{1, k_{o}, \downarrow}, \\
& \psi_{i} \approx \psi_{0, k_{i}, \downarrow}+\frac{\sqrt{2} \hbar \beta}{\ell_{B}\left(E_{c}-E_{z}\right)} \psi_{1, k_{i}, \uparrow},
\end{aligned}
$$

with the renormalized momenta obtained by solving $V_{c}\left(k_{o, i} \ell_{B}^{2}\right)-m^{*} \alpha^{2}(1 \pm 1) /\left(1+E_{z} / E_{c}\right)-m^{*} \beta^{2}(1 \mp 1) /(1-$ $\left.E_{z} / E_{c}\right) \mp E_{z} / 2=E_{F}-E_{c} / 2$, and the bulk gap is still dominated by the Zeeman energy. As a result the multiplicative factor in the square bracket Eq. (8) acquires 
an extra contribution $i U\left(V_{g}\right) \beta /\left(E_{c}-E_{z}\right)$ which adds in phase to the one controlled by $\delta \alpha\left(V_{g}\right)$ with positive sign providing a net further increase of the scattering amplitude.

\section{ROBUSTNESS TO DISORDER}

One of the major problems in the original Datta-Das transistor is the limitation to ballistic 2DEGs. Due to the spin-orbit interaction, the spin orientation is momentumdependent: elastic scattering randomizes the spin already after few scattering events spoiling the spin precession dynamics. A great advantage of our proposal is the overcoming of this problem. Indeed, the resonant nature of the spin-flip mechanism and the chiral, one-dimensional, character of integer $\mathrm{QH}$ edge states suggest that the resonant SFET is robust against disorder within the adiabatic transport regime 41 . To corroborate this intuitive argument we have run numerical tight-binding simulations which include: i) the presence of imperfections in the periodicity of the fingers, ii) the presence of corrugations at the sample edge. The results show deviations from the clean situation only for unrealistically strong disorder.

We now comment about two possible material systems (GaAs and InAs-based heterostructures), which suite best the implementation of our setup. Due to the larger Rashba coupling constant, a stronger effect could be seen in InAs-based devices. However, the larger Rashba coupling constant is also responsible for more sensitivity to disorder, resulting in a shorter spin-relaxation length $\ell_{\mathrm{sr}}$ that would limit the scalability of the device (the ratio being estimated as $\left.\ell_{\mathrm{sr}}^{\mathrm{InAs}} / \ell_{\mathrm{sr}}^{\mathrm{GaAs}} \sim 0.1^{25 / 39 / 40}\right)$. Besides, in InAs the $g$-factor can be about an order of magnitude larger than in GaAs. This implies a resonant periodicity of the array an order of magnitude smaller than the case of GaAs, which could represent a technical problem for very large $g$-factors.

\section{A. Imperfect periodic potential}

As a first kind of disorder we consider the case of nonperfect periodicity of the gate array potential. This class of disorder well describes imprecisions in the nanofabrication of the gates as well as non-uniformities in the different sections of the Hall bar. We model this disorder by randomizing each finger position in a range $\eta \lambda$ around its ideal position. The parameter $\eta$ can thus be considered as the "degree of imperfection": if $\eta=0 \%$ the array is ideal, whereas if $\eta \simeq 100 \%$ the position of each potential peak in the region is practically random, and the original periodicity is severely spoiled. In Fig. 4 (left panel) we present the curves of the transmission probability versus the applied top gate potential $U\left(V_{g}\right)$ for a device with 50 top gates and periodicity $\lambda=325 \mathrm{~nm}$, for different random realizations (shown in the right panel

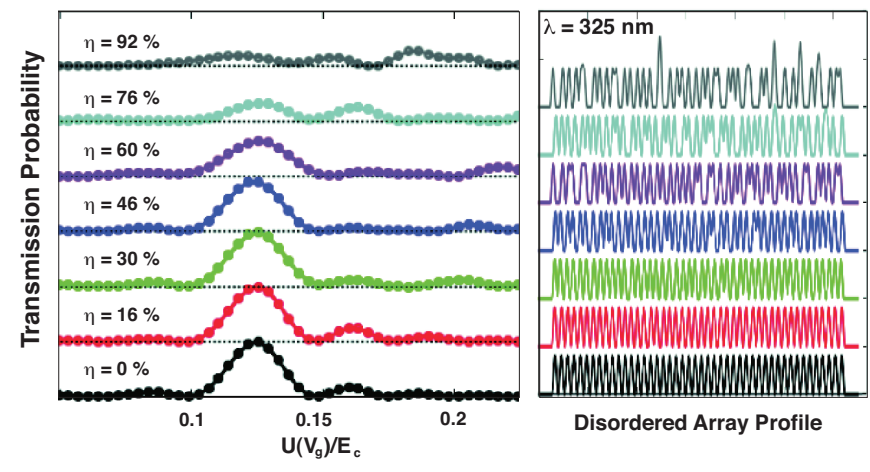

FIG. 4: (color online) Left Panel: plot of the transmission probability $\left|t_{i o}\right|^{2}$ versus applied gate potential $U\left(V_{g}\right)$ for a periodicity $\lambda=325 \mathrm{~nm}$, and different realizations of an imperfect periodic potential characterized by a degree of imperfection $\eta$. Each curve is relative to a different value of $\eta$ and is vertically shifted by 1: the maximum transmission is nearly unity up to $\eta<46 \%$. Right Panel: Profiles of the different realizations of imperfect periodic potential. All non-specified parameters are equal to the ones used for the simulations presented in Fig. 1 .

of Fig. 4). For degree of imperfection up to $\eta \sim 46 \%$ we see no difference in the position and height of the main resonance peak, although we record an attenuation of the secondary peaks as we increase the degree of imperfection. As we further increase the value of $\eta$ we see that the height of the main resonance peak gets lower and additional secondary peaks start to show up. This is due to the fact that the randomization procedure generates higher harmonics in the Fourier spectrum of the periodic potential, with reduced amplitude. The device response then corresponds to a sum of weak resonances at each harmonic. The results unequivocally show that the resonant SFET effect survives up to strong imperfections on the top gates positions.

\section{B. Disordered edges}

We now consider the possibility that the edges of the sample are "rough", i.e. not perfectly straight, and we study how such a roughness affects the functionality of the resonant SFET proposed. Rough edges are implemented through a random "corrugation" function (see Fig. 5 constructed with a random-walk procedure following a simple probability law. The latter is controlled by the parameter $p \in[0,1]$, which determines the characteristic length of the corrugation: for $p=1$ the profile is ideal, whereas for smaller $p$ it becomes progressively more corrugated. For $p=0$ the profile has a roughness on the scale of the lattice constant. This is illustrated in the right panel of Fig. 5, where six possible realizations of corrugated confining potentials, relative to as many values of $p$, are shown. Note that, for all the realizations considered, the transmission in the absence of a peri- 


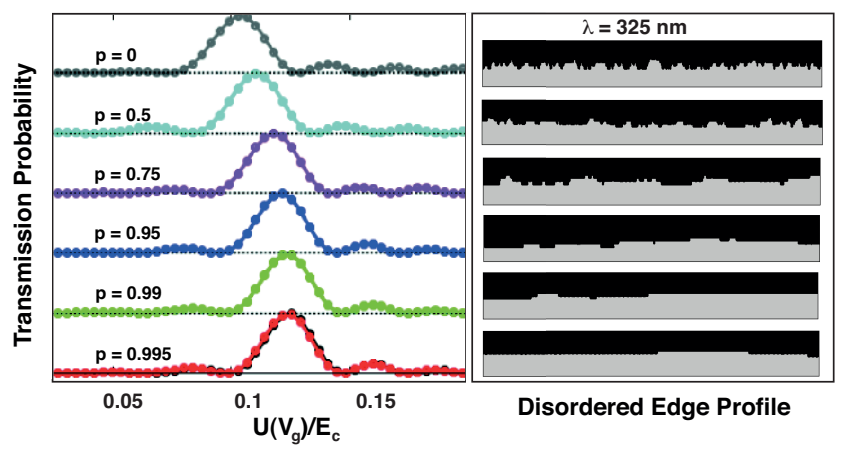

FIG. 5: (color online) Left Panel: plot of the transmission probability $\left|t_{i o}\right|^{2}$ versus applied gate potential $U\left(V_{g}\right)$ for a periodicity $\lambda=325 \mathrm{~nm}$, for different realizations of a rough confining potential characterized by the parameter $p$. Each curve is relative to a different value of $p$ and is vertically shifted by 1: for all the cases the maximum transmission reaches unity. A black curve for the case $p=1$, corresponding to the case of no disorder in the edge profile, is superimposed to the one for $p=0.995$. Right Panel: Profiles of the different realizations of rough confining potential. All non-specified parameters are equal to the ones used for the simulations presented in Fig. 1

odic array is negligible $(<0.05 \%)$. In the left panel of Fig. 5 we show the corresponding response of the device. For all disordered potential profiles considered we obtain a well defined resonant transmission, with a main resonance peak that reaches unity for suitable gate voltage $V_{g}$, and a series of lateral secondary peaks. We note, however, a progressive shift of the resonance peak to lower values of the gate potential $U\left(V_{g}\right)$, in correlation with an increase of the roughness of the edge profile and a consequent increase of the curvilinear abscissa of the edge channel between pairs of top gates. Such a shift of the resonance can be understood as an effective increase of the potential periodicity $\lambda$ that leads to a right-shift of the vertical cut in Fig. 2 of the main text. We can conclude that disorder related to corrugated edges does not spoil the resonant character of the device, but at most moves the resonance.

\section{CONCLUSIONS}

In this paper, a resonant SFET between chiral spinresolved edge states in the integer quantum Hall regime has been proposed. Coupling of the two spin states is obtained by applying an array of voltage-controlled top gates on the device. We notice that an analogous topgate technique has been recently shown to represent a valuable tool for measuring the strength of the Rashba SOI in a quantum wire with uniform Rashba SOI and no-magnetic field ${ }^{43}$. In our case the coupling mechanism arises as a resonant interplay between uniform and periodically modulated Rashba SOI that shows strong robustness against disorder in the system.

We acknowledge very useful conversations with $\mathrm{F}$. Ciccarello, M. Governale, B. Karmakar, G. La Rocca, V. Pellegrini, G. Usaj, and G. Vignale. This work was supported by MIUR through FIRB-IDEAS Project No. RBID08B3FM and EU-Projects IP-SOLID, STREPGEOMDISS, and NANOCTM.
${ }^{1}$ I. Žutić, J. Fabian, and S. Das Sarma, Rev. Mod. Phys. 76, 323 (2004); J. Fabian et al., Acta Physica Slovaca 57, 565 (2007).

2 D. Loss and D. P. DiVincenzo, Phys. Rev. A 57, 120 (1998).

3 S. Datta and B. Das, Appl. Phys. Lett 56, 665 (1990).

4 E. I. Rashba, Fiz. Tverd. Tela (Leningrad) 2, 1224 (1960) [Sov. Phys. Solid State 2, 1109 (1960)].

5 Y. A. Bychkov and E. I. Rashba, J. Phys. C 17, 6039 (1984).

6 J. Nitta, T. Akazaki, H. Takayanagi, and T. Enoki, Phys. Rev. Lett. 78, 1335 (1997).

7 J. B. Miller, D. M. Zumbühl, C. M. Marcus, Y. B. LyandaGeller, D. Goldhaber-Gordon, K. Campman, and A. C. Gossard, Phys. Rev. Lett. 90, 076807 (2003).

${ }^{8}$ L. Meier, G. Salis, I. Shorubalko, E. Gini, S. Schön, and K. Ensslin, Nat. Phys. 3, 650 (2007).

9 G. Schmidt, J. Phys. D: Appl. Phys. 38, R107 (2005).

10 S. A. Crooker, M. Furis, X. Lou, C. Adelmann, D. L. Smith, C. J. Palmstrøm, and P. A. Crowell, Science 309, 2191 (2005); I. Appelbaum, B. Huang, D. J. Monsma, Nature 447, 295 (2007); X. Lou, C. Adelmann, S. A. Crooker, E. S. Garlid, J. Zhang, K. S. Madhukar Reddy, S. D. Flexner, C. J. Palmstrøm, and P. A. Crowell, Nat. Phys.
3, 197 (2007); H. C. Koo, H. Yi, J.-B. Ko, J. Chang, S.-H. Han, D. Jung, S.-G. Huh, and J. Eom, Appl. Phys. Lett. 90, 022101 (2007).

11 H. C. Koo, J. H. Kwon, J. Eom, J. Chang, S. H. Han, and M. Johnson, Science 325, 1515 (2009).

12 S. Datta, Electronic Transport in Mesoscopic Systems (Cambridge UK, Cambridge University Press, 1997).

13 Y. Ji, Y. Chung, D. Sprinzak, M. Heiblum, D. Mahalu, Nature 422, 415 (2003); I. Neder, N. Ofek, Y. Chung, M. Heiblum, D. Mahalu, Nature 448, 333 (2007); I. Neder, M. Heiblum, Y. Levinson, D. Mahalu, and V. Umansky Phys. Rev. Lett. 96, 016804, (2006); I. Neder, M. Heiblum, D. Mahalu, and V. Umansky, Phys. Rev. Lett. 98, 036803 (2007).

14 P. Roulleau, F. Portier, D. C. Glattli, P. Roche, A. Cavanna, G. Faini, U. Gennser, and D. Mailly , Phys. Rev. B 76, 161309(R) (2007); P. Roulleau, F. Portier, D. C. Glattli, P. Roche, A. Cavanna, G. Faini, U. Gennser, and D. Mailly, Phys. Rev. Lett. 100, 126802 (2008); ibid. 102, 236802 (2009).

15 L. V. Litvin, H.-P. Tranitz, W. Wegscheider, and C. Strunk, Phys. Rev. B 75, 033315 (2007); L. V. Litvin, A. Helzel, H.-P. Tranitz, W. Wegscheider, and C. Strunk Phys. Rev. B 78, 075303 (2008). 
16 V. Giovannetti, F. Taddei, D. Frustaglia, and R. Fazio, Phys. Rev. B 77, 155320 (2008).

17 A. Bertoni, P. Bordone, R. Brunetti, C. Jacoboni, and S. Reggiani, Phys. Rev. Lett. 84, 5912 (2000).

18 R. Ionicioiu, G. Amaratunga, and F. Udrea, Int. J. Mod. Phys. B 15, 125 (2001).

19 T. M. Stace, C. H. W. Barnes, and G. J. Milburn, Phys. Rev. Lett. 93, 126804 (2004).

${ }^{20}$ I. L. Chuang and Y. Yamamoto, Phys. Rev. A 52, 3489 (1995).

21 G. Müller, D. Weiss, A. V. Khaetskii, K. von Klitzing, S. Koch, H. Nickel, W. Schlapp, and R. Lösch, Phys. Rev. B 45, 3932 (1992); S. Komiyama, H. Hirai, M. Ohsawa, and Y. Matsuda, S. Sasa and T. Fujii, Phys. Rev. B 45, 11085 (1992).

22 B. Karmakar, D. Venturelli, L. Chirolli, F. Taddei, V. Giovannetti, R. Fazio, S. Roddaro, G. Biasiol, L. Sorba, V. Pellegrini, and F. Beltram, Phys. Rev. Lett. 107, 236804 (2011).

${ }^{23}$ L. P. Kouwenhoven, F. W. J. Hekking, B. J. van Wees, C. J. P. M. Harmans, C. E. Timmering, C. T. Foxon, Phys. Rev. Lett. 65, 361 (1990).

${ }^{24}$ R. Winkler, Spin-orbit Interaction Effects in TwoDimensional Electron and Hole systems (Springer-Verlag, Berlin, 2003).

25 M. G. Pala, M. Governale, U. Zülicke, and G. Iannaccone, Phys. Rev. B 71, 115306 (2005).

26 V. L. Grigoryan, A. Matos Abiague, and S. M. Badalyan, Phys. Rev. B 80, 165320 (2009); V. L. Grigoryan, Armenian Jour. of Phys. 3, 45 (2010).

27 A. Reynoso, Gonzalo Usaj, M. J. Sánchez, C. A. Balseiro, Phys. Rev. B 70, 235344 (2004).

28 J. Dempsey, B. Y. Gelfand, and B. I. Halperin, Phys. Rev. Lett. 70, 3639 (1993); L. Rijkels and G. E. W. Bauer, Phys. Rev. B 50, 8629 (1994).

29 In particular, similarly to what happens with G1 and G3, for strong enough values of $V_{g}$ it can locally lower the filling factor and back scatter the inner edge state $\psi_{i}$ to the lower side of the Hall bar in Fig. 1. However, as shown in [V. Ya. Demikhovskii and A. A. Perov, Phys. Rev. B 75, 205307 (2007)] for the case of a two-dimensional infinite periodic potential, weak SOI, and relatively large lattice period, the Hall conductances of magnetic subbands are the same as for spinless particle, as predicted in [D. J. Thouless, M. Kohmoto, M. P. Nightingale, and M. den Nijs, Phys. Rev. Lett. 49, 405 (1982)].

30 X. F. Wang, Phys. Rev. B 69, 035302 (2004).

31 G. I. Japaridze, H. Johannesson, and A. Ferraz, Phys. Rev. B 80, 041308(R) (2009); M. Malard, I. Grusha, G. I. Japaridze, H. Johannesson, Phys. Rev. B 84, 075466 (2011).

32 A. Ström, H. Johannesson, and G. I. Japaridze, Phys. Rev. Lett. 104, 256804 (2010).

33 M. Büttiker, Y. Imry, R. Landauer, and S. Pinhas, Phys. Rev. B 31, 6207 (1985); M. Büttiker, Phys. Rev. B 38, 9375 (1988); M. Büttiker, Phys. Rev. B 46, 12485 (1992).

34 M. Di Ventra, Electrical Transport in Nanoscale Systems, Cambridge University Press, Cambridge UK (2008).

35 K. Kazymyrenko and X. Waintal, Phys. Rev. B 77, 115119 (2008).

36 J.Ferry, S. Goodnick, J. Bird, Transport in Nanostructures, Cambridge University Press; 2 edition (2009).

37 D. S. Fisher, and P. A. Lee, Phys. Rev. B 23, 6851 (1981).

38 W. Desrat, F. Giazotto, V. Pellegrini, and F. Beltram, F. Capotondi, G. Biasiol, L. Sorba, D. K. Maude, Phys. Rev. B 69, 245324 (2004).

39 A. V. Khaetskii, Phys. Rev. B 45, 13777 (1992).

40 D. G. Polyakov, Phys. Rev. B 53, 15777 (1996).

41 D. Venturelli, V. Giovannetti, F. Taddei, R. Fazio, D. Feinberg, G. Usaj and C. A. Balseiro, Phys. Rev. B 83, 075315 (2011).

42 G. Dresselhaus, Phys. Rev. 100, 580 (1955).

43 G. Thorgilsson, J. C. Egues, D. Loss, and S. I. Erlingsson, Phys. Rev. B 85, 045306 (2012). 